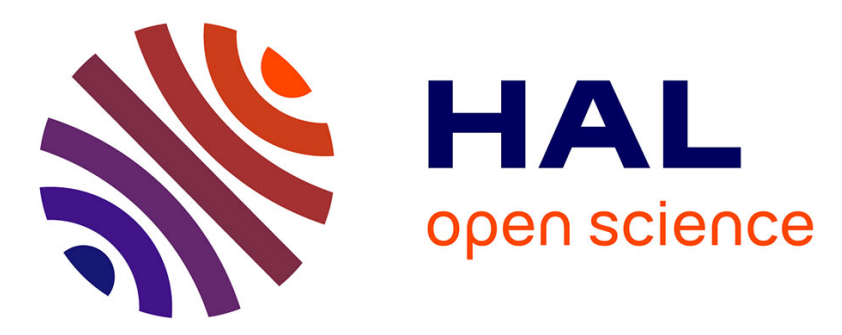

\title{
Relations entre la qualité du lait livré et les caractéristiques des exploitations
}

Claire Agabriel, J.B. Coulon, Gilles Brunschwig, C. Sibra, C. Nafidi

\section{To cite this version:}

Claire Agabriel, J.B. Coulon, Gilles Brunschwig, C. Sibra, C. Nafidi. Relations entre la qualité du lait livré et les caractéristiques des exploitations. Productions Animales, 1995, 8 (4), pp.251-258. hal-00896120

\section{HAL Id: hal-00896120 \\ https://hal.science/hal-00896120}

Submitted on 1 Jan 1995

HAL is a multi-disciplinary open access archive for the deposit and dissemination of scientific research documents, whether they are published or not. The documents may come from teaching and research institutions in France or abroad, or from public or private research centers.
L'archive ouverte pluridisciplinaire HAL, est destinée au dépôt et à la diffusion de documents scientifiques de niveau recherche, publiés ou non, émanant des établissements d'enseignement et de recherche français ou étrangers, des laboratoires publics ou privés. 
INRA Prod. Anim., $1995,8(4), 251-258$
C. AGABRIEL, J.B. COULON*,

G. BRUNSCHWIG, C. SIBRA,

C. NAFIDI**

ENITA Clermont-Ferrand, 63370 Lempdes

* INRA Laboratoire Adaptation des Herbivores aux

Milieux, Theix 63122 St Genès Champanelle

** ENIL, route de Salers, 15000 Aurillac

\section{Relations entre la qualité du lait livré et les caractéristiques des exploitations}

Le prix du lait payé aux éleveurs est fonction de sa composition - taux butyreux et protéique - et de sa qualité hygiénique. De nombreux éléments de la conduite du troupeau, tels que l'alimentation ou les pratiques de traite, interviennent simultanément sur ces paramètres. L'étude, dans les élevages, des relations entre l'évolution des critères de qualité et l'ensemble des facteurs qui les influencent permet de définir les améliorations à apporter à la conduite d'élevage.

De nombreuses études ont été réalisées sur les facteurs de variation de la qualité du lait livré à l'échelle de l'exploitation, en France ou à l'étranger. Elles ont été généralement conduites sur des exploitations ciblées (de par leur niveau de production, la race des animaux ou le suivi technique des troupeaux), et

\section{Résumé}

Cent-quarante trois exploitations laitières dans 2 zones du Massif Central ont fait l'objet d'une enquête détaillée concernant à la fois la structure de l'exploitation et du troupeau, l'utilisation des surfaces, les pratiques alimentaires hivernales et estivales, les pratiques de traite et l'hygiène des animaux. Parallèlement des caractéristiques du lait (taux butyreux et protéique, numération cellulaire, flore totale et contamination butyrique) ont été relevées mensuellement pendant 14 mois. Ces données ont permis d'analyser la variabilité des caractéristiques du lait en fonction des caractéristiques des exploitations. En dehors de l'effet de la race des vaches laitières, ce sont des variables de maîtrise de l'alimentation qui permettent le mieux d'expliquer les variations de la composition chimique du lait (nature du fourrage et niveau des apports nutritifs). La qualité bactériologique du lait a pu être associée à la fois à la nature des fourrages hivernaux (contamination butyrique) et à l'hygiène de traite. Dans la plupart des exploitations, la numération cellulaire a été sensiblement plus élevée en été qu'en hiver. Peu d'exploitations présentent à la fois un taux protéique élevé et des caractéristiques hygiéniques du lait optimales. Dans les 2 zones, c'est au cours de l'hiver que l'on observe la plus grande variabilité de composition du lait, liée aux différences de conduite alimentaire hivernale. D'une manière générale, l'hygiène du lait reste peu maîtrisée dans un grand nombre de troupeaux, et, dans la majorité des exploitations de la zone Nord, l'alimentation hivernale semble insuffisante pour assurer l'expression d'un taux protéique élevé. n'ont concerné que certains des éléments de la qualité du lait, en général sa composition chimique (Coulon et Binet 1987, Seegers et al 1989, Agabriel et al 1990 et 1993, GVL 1993, Dobbelaar et al 1994). Si les différentes caractéristiques du lait prises en compte dans le paiement aux éleveurs et retenues dans cette étude (taux butyreux et protéique, numération cellulaire, teneur en spores butyriques et flore totale) dépendent beaucoup des facteurs du milieu, ces facteurs sont différents selon les caractéristiques considérées. Leur étude nécessite la prise en compte conjointe de la conduite alimentaire des animaux (Hoden et Coulon 1991) et de leur hygiène, en particulier au cours de la traite (Serieys 1992, Hartheiser 1994, Monsallier 1994). Cette approche est particulièrement importante dans les régions comme l'Auvergne, où une partie importante du lait est transformée en fromages d'Appellation d'Origine Contrôlée (AOC) pour lesquels les conditions de production du lait et ses caractéristiques chimiques et bactériologiques revêtent une importance particulière.

L'objectif de cette étude est d'analyser les relations entre certaines caractéristiques des exploitations et l'ensemble des critères de qualité de leur lait. Elle s'inscrit dans l'étude générale, conduite dans le cadre du Pôle AOC Massif Central (Ballot et Lilas 1994), des systèmes d'exploitations en zone AOC du Massif Central. 


\section{1 / Conduite de l'étude}

\section{Echantillonnage}

Cette étude a été effectuée à partir des données d'analyse du lait (paiement à la qualité) et d'enquêtes effectuées dans des exploitations du Cantal et du Puy-de-Dôme. Deux zones naturelles ont été définies: une zone Sud d'altitude modérée ( 400 à $700 \mathrm{~m}$ ) où la culture du maiis est possible, située dans la Châtaigneraie et le bassin d'Aurillac, et une zone Nord plus élevée, correspondant à une partie du Cézallier-Sancy et des Monts du Cantal. Quatre-vingt-dix exploitations ont été choisies dans chaque zone, soit environ $10 \%$ du total. Ces exploitations étaient représentatives des principales laiteries et de l'ensemble de la gamme des niveaux de livraison. Parmi ces 180 exploitations, 143 ont fait l'objet d'enquêtes pendant l'automne 1993: 61 dans la Zone Nord et 82 dans la zone Sud. Sur chacune des exploitations enquêtées, les données recueillies ont concerné d'une part leur histoire, leur structure, l'utilisation des surfaces, la main d'œuvre et la présence d'autres ateliers d'élevage, et d'autre part la conduite du troupeau laitier (logement, traite, répartition des vêlages, alimentation hivernale et estivale, état sanitaire). Par ailleurs, les quantités de lait livré et ses caractéristiques mensuelles (taux butyreux et protéique, flore totale, nombre de cellules et de spores butyriques) ont été relevées à l'échelle du troupeau entre août 1992 et septembre 1993 (données des laiteries, moyenne de deux à trois prélèvements par mois).

\section{Traitement des données}

A partir de l'examen des évolutions moyennes des caractéristiques du lait au

Tableau 1. Caractéristiques des exploitations selon la zone.

\begin{tabular}{|c|c|c|}
\hline Zone & Nord & Sud \\
\hline Nombre d'exploitations & 61 & 82 \\
\hline SAU (ha) & 66 & 38 \\
\hline dont prairies permanentes (ha) & 52 & 10 \\
\hline Nombre de vaches laitières & 29 & 28 \\
\hline \multicolumn{3}{|l|}{ Production laitière } \\
\hline - lait livré (1 000 l) & 90 & 131 \\
\hline \multirow[t]{2}{*}{ - lait/vache/an (l) } & 3515 & 4640 \\
\hline & \multicolumn{2}{|c|}{ \% d'exploitations } \\
\hline Vente directe de lait ou fromage & 15 & 0 \\
\hline Race Prim'Holstein dominante & 26 & 61 \\
\hline Stabulation entravée & 94 & 58 \\
\hline Traite manuelle & 16 & 0 \\
\hline \multicolumn{3}{|l|}{ Alimentation hivernale } \\
\hline - foin dominant & 72 & 4 \\
\hline - présence d'ensilage de maïs & 0 & 85 \\
\hline \multicolumn{3}{|l|}{ Alimentation estivale } \\
\hline - pâturage rationné & 57 & 89 \\
\hline - apport de fourrage conservé & 7 & 79 \\
\hline - apport de concentré & 69 & 96 \\
\hline \multicolumn{3}{|l|}{ Période de vêlage } \\
\hline - août à novembre & 16 & 33 \\
\hline - décembre à mars & 51 & 13 \\
\hline
\end{tabular}

cours de l'année, deux périodes représentatives de l'hiver (moyennes des mois de janvier, février et mars) et de l'été (moyennes des mois de juillet, août et septembre) ont été retenues.

Une typologie des exploitations de l'ensemble des 2 zones a été réalisée à l'aide d'une classification ascendante hiérarchique construite à partir des résultats d'une analyse factorielle des correspondances (AFC). Cette $\mathrm{AFC}$ a été réalisée à partir des caractéristiques du lait au cours des 2 périodes définies ci-dessus. Les caractéristiques du lait ont été découpées en classes de la manière suivante: pour les taux butyreux et protéique, à partir de la moyenne observée sur chaque période, de manière à avoir des classes équilibrées ; pour la numération cellulaire, les exploitations présentant, pour chaque période, 3 mois inférieurs à 300000 cellules/ml ont été affectées à la classe 1 , celles présentant 2 ou 3 mois sur 3 supérieurs à 400000 cellules $/ \mathrm{ml}$ ont été affectées à la classe 3 , et les autres à la classe 2 . La même méthode a été adoptée pour la teneur en spores butyriques et la flore totale, ce qui donne pour la classe 1 : inférieur à 500 spores $/ 1$ ou 50000 germes/ml pendant 3 mois; et pour la classe 3 : supérieur à 1000 spores/1 ou 50000 germes $/ \mathrm{ml}$ pendant 2 ou 3 mois sur 3 . Compte tenu de l'absence de certaines données de qualité du lait dans 6 exploitations, cette typologie n'a été réalisée que sur 137 exploitations.

\section{2 / Résultats}

\section{1 / Caractéristiques générales des exploitations}

La plupart des caractéristiques du milieu ont été très différentes entre le Nord et le Sud (tableau 1). Il s'agit d'abord des variables de structure : altitude (respectivement 1051 et $598 \mathrm{~m}$ pour le Nord et le Sud), taille des exploitations (respectivement 66 et 38 ha pour le Nord et le Sud), âge et formation des éleveurs, quota (respectivement 85000 et 128000 1), type de bâtiment (respectivement $94 \%$ et $58 \%$ de stabulations entravées dans le Nord et le Sud)... Par ailleurs, l'utilisation des surfaces (respectivement 79 et $26 \%$ de prairies permanentes), leur mode de récolte (respectivement 26 et $94 \%$ des exploitations récoltent le fourrage sous forme ensilée ou enrubannée), les caractéristiques des ateliers animaux (respectivement 48 et $35 \%$ des éleveurs possèdent des vaches allaitantes) et les pratiques de conduite du troupeau laitier ont aussi été très différents. En particulier, les vaches laitières, dont la race est beaucoup plus variable dans le Nord que dans le Sud, ont vêlé plutôt en hiver dans le Nord et plutôt en automne dans le Sud. Les $3 / 4$ des troupeaux du Sud reçoivent de l'ensilage de maïs pendant l'hiver (0\% dans le Nord) alors que $72 \%$ des rations de base hivernales des trou- 
peaux du Nord sont constituées exclusivement de foin (4\% dans le Sud). Celui-ci était généralement stocké en petites bottes dans le Nord ( $75 \%$ des exploitations), et en balles rondes dans le Sud ( $84 \%$ des exploitations). La conduite du pâturage a aussi été différente (pâturage rationné dans respectivement 57 et $89 \%$ des troupeaux du Nord et du Sud, et complémenté par du fourrage conservé dans respectivement 7 et $79 \%$ des cas).

Les caractéristiques du lait ont été très variables d'un mois à l'autre (figure 1) et d'une exploitation à l'autre, dans le Nord comme dans le Sud. En moyenne le taux butyreux a été supérieur et plus stable au cours de l'année dans le Sud que dans le Nord, où il a été nettement plus faible en fin d'hiver : à cette période, l'écart moyen entre les 2 zones atteint près de $5 \mathrm{~g} / \mathrm{l}$. Le taux pro-

Figure 1. Evolution des caractéristiques du lait au cours de l'année selon la zone.
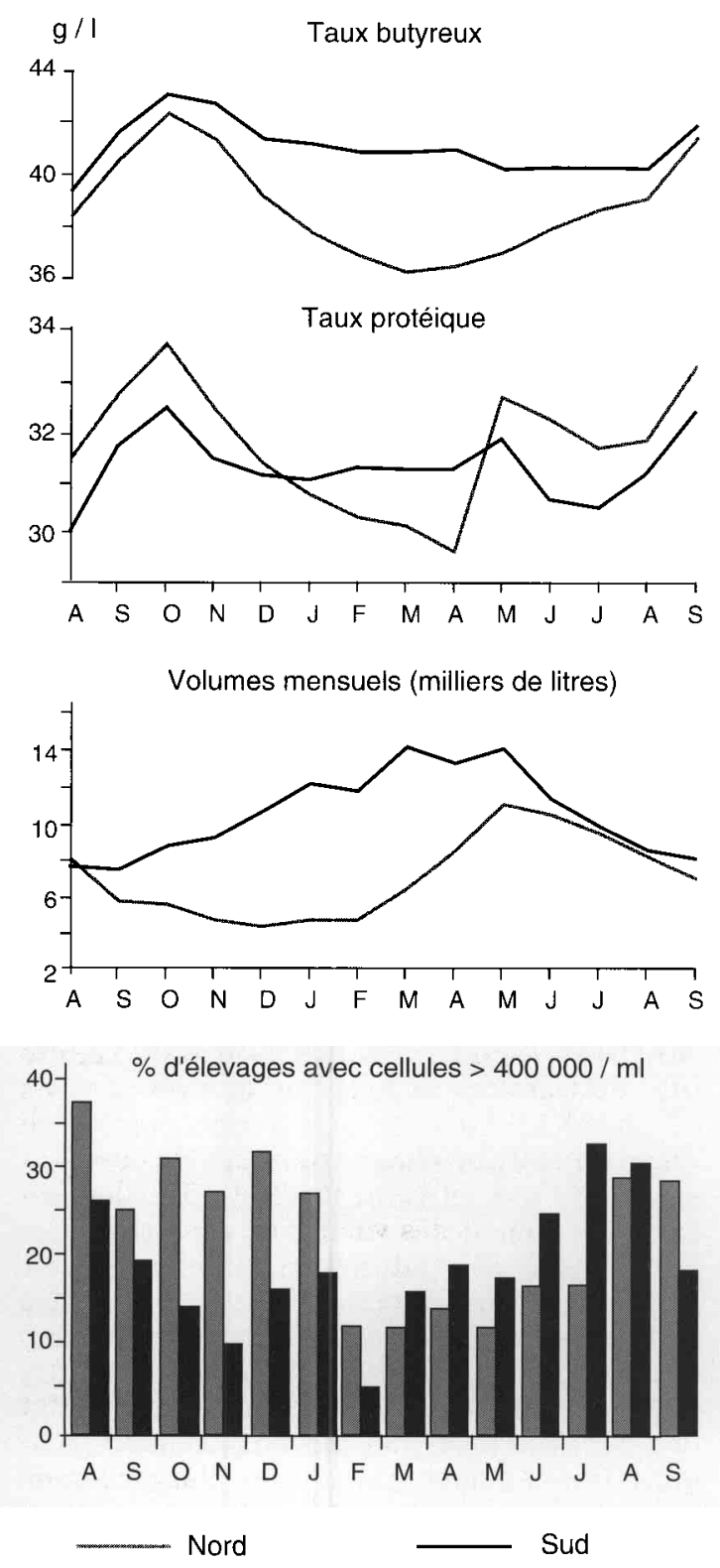

téique a été plus élevé l'hiver dans le Sud que dans le Nord. Cet écart s'est inversé l'été en raison de l'effet favorable très important de la mise à l'herbe dans le Nord $(+3 \mathrm{~g} / \mathrm{l}$ entre avril et mai, contre $+0,5 \mathrm{~g} / \mathrm{l}$ dans le Sud). En moyenne, la flore totale a été plus élevée dans le Nord où environ $35 \%$ des prélèvements contenaient plus de 50000 germes $/ \mathrm{ml}$ (contre $8 \%$ dans le Sud). La teneur en spores butyriques a été voisine dans les 2 zones. Elle a augmenté dès la rentrée à l'étable pour atteindre en fin d'hiver ses valeurs maximales (respectivement 30 et $46 \%$ des prélèvements de février et mars à plus de 1000 spores/l, pour le Nord et le Sud). A l'inverse, les taux de cellules ont été les plus faibles en fin d'hiver (respectivement 12 et $4 \%$ des prélèvements à plus de 400000 cellules $/ \mathrm{ml}$ pour le Nord et le Sud) et les plus élevés en fin d'été (figure 1). Les livraisons mensuelles ont été maximales en fin d'hiver dans le Sud et au printemps dans le Nord.

\section{2 / Typologie des exploitations d'après les caractéristiques du lait}

La classification des exploitations sur la base des caractéristiques de leur lait a permis de définir 5 classes principales (tableau 2 et figure 2 ). La première $(\mathbf{n}=38$ ) regroupe des exploitations dont le lait présente des taux de matières grasses et de protéines élevés (respectivement 42,6 et $31,9 \mathrm{~g} / \mathrm{l}$ à l'échelle de l'année) et réguliers au cours de l'année. Ces laits sont aussi les plus propres et les moins chargés en cellules et en spores butyriques. Ces exploitations disposent d'animaux principalement de type Pie-noir (26/38), d'un niveau de production élevé (environ $5300 \mathrm{l} / \mathrm{vache} / \mathrm{an}$ ), vêlant en automne ou de manière étalée sur l'année et alimentés l'hiver essentiellement avec des rations où l'ensilage de maïs est très généralement présent (32/38), le plus souvent de manière dominante dans la ration $(22 / 38)$. L'été les animaux sont conduits en pâturage rationné ou tournant et souvent complémentés avec des fourrages conservés (34/38) et des aliments concentrés (34/38). La traite est généralement réalisée en salle de traite (22/38) et son hygiène est bonne (21 exploitations sur 38 ont une note supérieure ou égale à 7). La grande majorité pratique un tarissement brutal (32/38). La quasi-totalité de ces exploitations (35 sur 38) sont situées dans la zone Sud.

La deuxième classe $(\mathbf{n}=\mathbf{3 1})$ est caractérisée par des taux butyreux et protéique faibles en hiver (respectivement 37,0 et $30,2 \mathrm{~g} / \mathrm{l})$, mais assez élevés en été, la mise à l'herbe apparaissant comme une période de variation considérable du taux protéique du lait $(+2,7 \mathrm{~g} / \mathrm{l}$ entre avril et mai). L'hiver, les teneurs en spores butyriques sont en général élevées, de même que la flore totale et les numérations cellulaires (celles-ci sont également élevées en été). Les animaux de ces exploitations sont de race Salers (9/31), Montbéliarde $(6 / 31)$ ou de type Pie-noir $(12 / 31)$ et 
Figure 2. Evolution des caractéristiques du lait au cours de l'année selon les classes de qualité du lait.
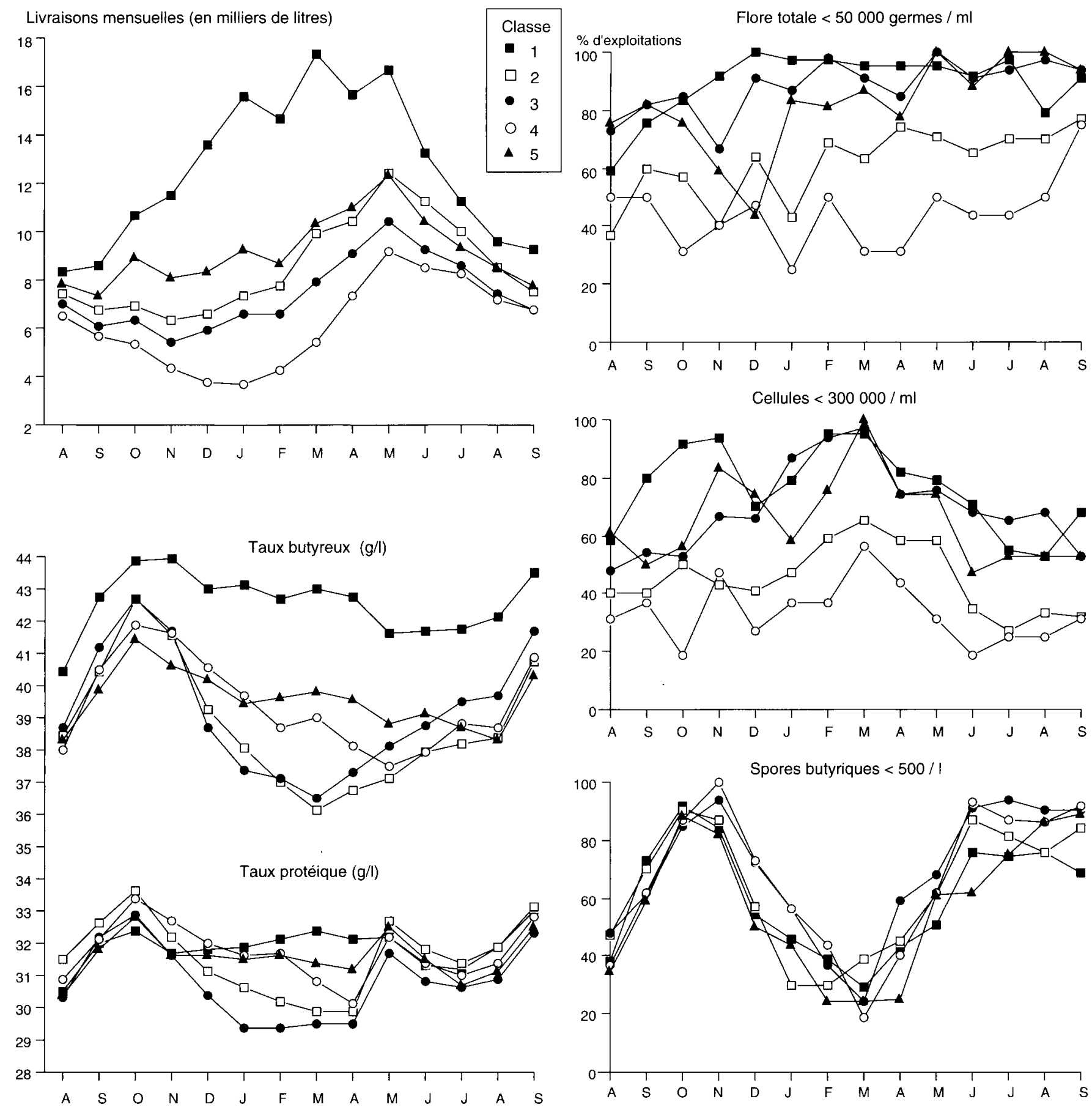

La composition du

lait est d'abord

liée à la race des vaches puis à leur conduite alimentaire : nature du fourrage et niveau des apports. sont alimentés l'hiver avec des rations à base de foin (17/31) ou d'ensilage d'herbe, parfois associés à un peu d'ensilage de maïs. Pour $1 / 3$ des exploitations, la complémentation hivernale n'est pas raisonnée en fonction du niveau de production des vaches. L'été, c'est dans cette classe que la pratique du pâturage libre est la plus fréquente (7/31). Au pâturage, la majorité des exploitations distribuent de l'aliment concentré $(25 / 31)$, mais peu offrent des fourrages conservés (10/31). La production individuelle est en moyenne faible (environ $3800 \mathrm{l} / \mathrm{an}$ ). L'hygiène de traite est mauvaise (20 exploitations sur 31 ont une note inférieure à 7). Ces exploitations se ren- contrent aussi bien dans la zone Nord $(\mathrm{n}=18)$ que dans la zone Sud $(\mathrm{n}=13)$.

La troisième classe $(n=34)$ est caractérisée par des taux butyreux et protéique faibles, en particulier l'hiver (respectivement 36,7 et $29,6 \mathrm{~g} / \mathrm{l}$ ), une forte contamination en spores butyriques, mais des numérations cellulaires et une flore totale très faibles. Comme dans la classe 2 , le taux protéique augmente fortement à la mise à l'herbe $(+2,2 \mathrm{~g} /$ entre avril et mai). Les caractéristiques de ces exploitations (race, ration hivernale et conduite estivale, niveau de production) sont très voisines de celles de la classe 2 . 
Tableau 2. Caractéristiques des différentes classes d'exploitations ${ }^{(1)}$ constituées à partir de la qualité du lait.

\begin{tabular}{|c|c|c|c|c|c|c|}
\hline Classes & 1 & 2 & 3 & 4 & 5 & \\
\hline Nombre d'exploitations (Nord + Sud) & $3+35$ & $18+13$ & $18+16$ & $11+5$ & $7+11$ & \\
\hline Caractéristiques du lait(2) & & & & & & \\
\hline Taux butyreux (g/l) & 43,0 & 37,0 & 36,7 & 39,1 & 39,5 & $* *$ \\
\hline Taux protéique (g/l) & 32,1 & 30,2 & 29,6 & 31,4 & 31,5 & $* *$ \\
\hline Butyriques $(<500 \text { spores } /)^{(3)}$ & 13 & 3 & 7 & 3 & 5 & $* *$ \\
\hline Cellules $\left.(<300000 / \mathrm{ml})^{3}\right)$ & 28 & 10 & 28 & 5 & 10 & $* *$ \\
\hline Flore totale $(<50000 / \mathrm{ml})^{\{3\}}$ & 34 & 4 & 29 & 1 & 14 & $* *$ \\
\hline Nombre de vaches laitieres & 30 & 31 & 26 & 22 & 25 & + \\
\hline Production laitière (l/vache/an) & 5298 & 3770 & 3518 & 3775 & 4206 & $* *$ \\
\hline & & \multicolumn{4}{|c|}{ Nombre d'exploitations dans chaque classe } & \\
\hline Race dominante & & & & & & $* *$ \\
\hline Prim'Holstein & 26 & 12 & 14 & 5 & 4 & \\
\hline Montbéliarde ou Abondance & 8 & 6 & 8 & 7 & 10 & \\
\hline Salers & 0 & 9 & 3 & 1 & 0 & \\
\hline Troupeau mixte ${ }^{(4)}$ & 4 & 4 & 9 & 3 & 4 & \\
\hline Ration de base hivernale & & & & & & $* *$ \\
\hline Foin & 3 & 17 & 18 & 10 & 10 & \\
\hline Ensilage d'herbe + foin & 3 & 5 & 6 & 2 & 1 & \\
\hline Ensilage de maïs dominant & 22 & 6 & 6 & 3 & 5 & \\
\hline Ensilage de maïs non dominant ${ }^{(5)}$ & 10 & 3 & 4 & 1 & 2 & \\
\hline Alimentation estivale & & & & & & \\
\hline Apport de fourrage conservé & 34 & 10 & 11 & 3 & 11 & ** \\
\hline Apport de concentré & 34 & 25 & 28 & 9 & 16 & $* *$ \\
\hline Installation de traite & & & & & & $* *$ \\
\hline Salle de traite & 22 & 7 & 2 & 2 & 0 & \\
\hline Pots & 4 & 7 & 21 & 11 & 8 & \\
\hline Note de préparation à la traite $\geqslant 7$ & 21 & 11 & 13 & 5 & 7 & $\mathrm{~ns}$ \\
\hline Période de vêlage dominante & & & & & & $* *$ \\
\hline Août à novembre & 21 & 8 & 11 & 1 & 5 & \\
\hline Décembre à mars & 2 & 12 & 12 & 10 & 4 & \\
\hline Etalée & 15 & 11 & 11 & 5 & 9 & \\
\hline
\end{tabular}

(1) Le détail de l'ensemble des variables enquêtées et de leur traitement est disponible auprès des auteurs.

(2) Au cours des mois de janvier, février et mars.

(3) Nombre d'exploitations.

(4) Vaches à la fois allaitantes et traites.

(5) Ou alterné avec ensilage d'herbe.

Les classes 4 et 5 (respectivement $\mathbf{n}=16$ et $\mathbf{n}=18$ ) présentent des laits de composition chimique voisine (taux butyreux et protéique de respectivement 39,5 et $31,6 \mathrm{~g} / \mathrm{l}$ à l'échelle de l'année), et très souvent contaminés en spores butyriques. Elles se distinguent par la teneur en germes totaux et par la numération cellulaire estivale, très élevées dans la classe 4 et faibles dans la classe 5 . Dans ces 2 classes, les vaches sont généralement de race Montbéliarde (17/34) ou de type Pie-noir (9/34), et produisent environ $4000 \mathrm{l}$ de lait/an. L'hiver, l'alimentation est à base d'herbe sous forme de foin ou d'ensilage (23/34). La complémentation hivernale est plus adaptée aux besoins des vaches dans la classe 5 (17/18) que dans la classe 4 (8/16), de même que la complémentation au pâturage, en fourrage conservé (respectivement 11/18 et $3 / 16$ ) et en concentré (respectivement 16/18 et 9/16). Dans ces 2 classes, l'hygiène de traite est médiocre (12 exploitations seulement présentent une note supérieure ou égale à 7 ). Les vêlages ont lieu l'hiver dans la classe 4 et de manière étalée dans la classe 5 .

\section{Discussion}

La variabilité de la composition chimique du lait d'une exploitation à l'autre a été considérable, supérieure à celle observée dans d'autres enquêtes (Agabriel et al 1993) même lorsqu'elles étaient faites sur un échantillon représentatif (Coulon et al 1988), vraisemblablement en raison de la diversité des facteurs de variation présents (race, nature et niveau d'alimentation...). En ce qui concerne le taux butyreux, cette variabilité est ainsi sensiblement réduite lorsqu'on la calcule intra zone. En effet, une grande partie de l'écart de taux butyreux entre les 2 zones, en faveur du Sud, est liée à la présence d'ensilage de mais dans la plupart des exploitations de cette zone, dont l'effet spécifique sur le taux butyreux est bien connu (Hoden et Coulon 1991). Cet écart est accentué par la présence, dans la zone Nord, de nombreux troupeaux de vaches Pierouge et par l'existence de systèmes de type traditionnel Salers (12 exploitations) où la traite partielle des animaux conduit à récolter un lait plus pauvre en matières grasses. Une autre différence apparaît entre les 2 zones
La qualité hygiénique du lait est liée à la nature des fourrages hivernaux (ensilage, foin) et aux pratiques de traite. 
sur l'évolution du taux protéique du lait au cours de l'année : dans toutes les exploitations de la zone Nord, la mise à l'herbe s'accompagne d'une augmentation considérable du taux protéique $(+3 \mathrm{~g} / \mathrm{l}$ en moyenne entre les mois d'avril et de mai), témoin d'une alimentation hivernale déficiente, en particulier du point de vue énergétique. L'augmentation du taux protéique à la mise à l'herbe est en effet un bon indicateur de la conduite alimentaire hivernale (Coulon 1991). Ceci met en évidence une marge de progrès considérable dans l'ensemble de ces exploitations, d'autant que ces taux les plus faibles apparaissent à une période où les quantités de lait livré sont relativement importantes. Par ailleurs, dans les 2 zones, on observe rarement des laits à la fois pauvres en spores butyriques, en cellules et en flore totale (15 exploitations sur les 137 présentent, sur les 3 mois d'hiver, à la fois moins de 500 spores $/ 1$, moins de 300000 cellules $/ \mathrm{ml}$ et moins de 50000 germes $/ \mathrm{ml}$ ). Ces 15 exploitations se trouvent dans les 2 zones (respectivement 6 et 9 dans les zones Nord et Sud), et présentent des caractéristiques très variables, en particulier en ce qui concerne la race des animaux (Holstein, Montbéliarde et Salers), les effectifs (10 à 60), et les niveaux de production par vache (de moins de 1000 à plus de 8000 l/an). Elles diffêrent cependant des autres exploitations par la moindre présence de l'ensilage d'herbe dans la ration hivernale ( $1 / 3$ de ces exploitations utilisent de l'ensilage d'herbe contre $2 / 3$ dans le reste de l'échantillon). Par ailleurs, et de manière étonnante, la plupart de ces exploitations présentent une note de traite inférieure à 7 , mais la qualité du nettoyage du matériel de traite et de stockage du lait semble légèrement meilleure.

Dans le Sud, la période estivale apparaît problématique pour les cellules. L'augmentation saisonnière de la numération cellulaire a déjà été observée dans d'autres situations (Coulon et Lilas 1988, Coulon et al 1988, Dubeuf 1995), sans que les raisons soient clairement mises en évidence (effet indirect lié à l'avancement du stade de lactation lorsque les animaux ont vêlé en début d'hiver, moindre attention portée aux animaux à cette période, prolifération des mouches...). Il faut cependant remarquer que la numération cellulaire observé sur le lait de tank est autant une mesure de la rigueur du tri des laits mammiteux réalisé par l'éleveur que de la fréquence intrinsèque des mammites dans l'exploitation. Il est possible que ce tri du lait soit différent d'une exploitation à l'autre et, au sein d'une même exploitation, d'une saison à l'autre.

Au-delà de ces constatations générales, l'analyse détaillée des caractéristiques du lait a permis de confirmer :

1) l'importance conjointe des facteurs génétiques (race des vaches) et alimentaires (nature de la ration pour le taux butyreux et niveau des apports énergétiques pour le taux protéique) sur la composition chimique du lait (Journet et Chilliard 1985, Rémond
1985). L'analyse des évolutions des taux butyreux et protéique au cours de l'année permet d'illustrer les effets cumulatifs ou au contraire antagonistes de ces 2 grands types de facteurs (figure 2) : ainsi la classe 1 présente des taux butyreux constamment supérieurs à ceux des autres classes, en raison du type de vache (Pie-noir) prépondérant dans cette classe (qui explique vraisemblablement l'essentiel de l'écart estival), et de la présence quasi systématique d'ensilage de maiis dans la ration hivernale qui conduit aux écarts les plus élevés au cours de l'hiver. Le taux protéique au contraire n'est supérieur dans cette classe qu'au cours de l'hiver, en raison de l'effet favorable de l'alimentation qui fait plus que compenser l'effet défavorable de la race. En été, le taux protéique des troupeaux de la classe 1 est généralement inférieur à celui de la classe 2, où les animaux de type Pie-rouge prédominent. Enfin, il est intéressant de remarquer que les classes 4 et 5 , qui présentent des taux butyreux et protéique identiques à l'échelle de l'année, présentent une évolution de ces taux différente (beaucoup plus régulière dans la classe 5 que dans la classe 4) certainement due à la répartition différente des vêlages dans ces 2 classes (étalée dans la classe 5, plutôt regroupée en hiver dans la classe 4).

2) l'importance de l'hygiène, et en particulier de l'hygiène de traite sur la numération cellulaire du lait (Badinand 1994, Dubeuf 1995). Dans cette étude, lorsque la note de traite est inférieure à 9, $31 \%$ des exploitations présentent des prélèvements hivernaux dont la numération cellulaire est supérieure à 300000 cellules $/ \mathrm{ml}$ contre seulement $10 \%$ lorsque cette note est supérieure ou égale à 9 (figure 3). Cette note est cependant peu liée à la flore totale et à la teneur en spores butyriques (figure 3 ) en raison d'une part du caractère incomplet de cet indicateur (la note de traite ne prend en compte que les pratiques de préparation à la traite) et d'autre part de l'existence d'autres facteurs de risques situés en amont (contamination des fourrages, propreté des animaux) ou en aval (entretien du matériel de traite et de stockage du lait).

\section{Conclusion}

Cette étude montre qu'il demeure une marge de progrès importante en matière de qualité du lait dans les exploitations enquêtées qui étaient représentatives de l'ensemble de celles des 2 zones choisies (cas du taux protéique dans la zone Nord, et de la qualité hygiénique d'une manière générale). Comme cela a été récemment mis en évidence dans une étude réalisée dans les Alpes du Nord (Dubeuf 1995), rares sont les exploitations où l'ensemble des facteurs favorables à la fois à un lait riche en matières utiles et de bonne qualité hygiénique sont réunis. D'un point de vue pratique, il est nécessaire de hiérarchiser les possibilités d'amélioration selon leur diffi- 
culté de mise en œuvre, leur délai de réponse et leur coût. Par exemple, l'amélioration de la qualité bactériologique et des numérations cellulaires doit pouvoir être réalisée par l'application de règles d'hygiène bien connues et de traitement des animaux (au tarissement) ne remettant pas en cause le fonctionnement de l'exploitation. Par ailleurs, les contaminations butyriques élevées dans les exploitations de la zone Nord en système foin, dues essentiellement à la présence importante de terre dans les foins liée à la prolifération des rats taupiers ces dernières années dans cette zone, peuvent être considérées comme conjoncturelles. Il n'en va pas de même lorsque ces contaminations sont observées dans des exploitations où l'alimentation hivernale est à base d'ensilage d'herbe, milieu privilégié de développement des spores butyriques lorsque l'acidification du silo est insuffisante. D'un point de vue technique, l'amélioration de la teneur en protéines et la diminution de la contamination en spores butyriques des laits de troupeaux alimentés avec des rations hivernales à base d'ensilage d'herbe est possible sans remettre forcément en cause le système fourrager. Cette amélioration passe d'abord par celle (peu coûteuse mais contraignante) des conditions de récolte et de conservation des ensilages, dont l'effet se répercutera à la fois sur la contamination butyrique et sur leur ingestibilité et leur valeur azotée (Demarquilly et Andrieu 1988). Dans ces conditions, il est possible d'obtenir des laits très peu contaminés en spores butyriques (Coulon et al 1991) et des performances de production voisines de celles obtenues avec d'autres fourrages (Coulon et al 1995). La modification du système fourrager est une autre alternative, beaucoup plus contraignante à mettre en œuvre et dont les conséquences sur le fonctionnement de l'exploitation (récolte des fourrages, valeur alimentaire des rations, gestion du pâturage...) dépassent largement le cadre de la qualité du lait.

Ce travail doit faire l'objet d'une poursuite dans 2 voies :

- approfondissement de la caractérisation des systèmes de production, via l'étude complémentaire des paramètres socio-économiques,

- approfondissement de la caractérisation de la qualité bactériologique du lait, dans le cadre d'une production de fromage d'AOC au lait cru. Une vingtaine d'exploitations parmi les 143 ayant fait l'objet de cette première étude font actuellement l'objet d'un suivi régulier, axé essentiellement sur la composition de la flore du lait, de manière à mettre en évidence d'éventuels facteurs de variations de cette flore liés à la conduite du troupeau.

\section{Remerciements}

Nous tenons à remercier les éleveurs qui ont participé à l'enquête et les étudiants de l'ENITA de ClermontFerrand et de l'ENIL d'Aurillac qui l'ont réalisée, ainsi que les responsables des entreprises laitières qui ont permis sa mise en œuvre.
Figure 3. Relation entre les caractéristiques hygiéniques du lait (moyenne des mesures hivernales réalisées au cours des mois de janvier, février et mars) et la note de traite.

L'hygiène de traite a été appréhendée par un indicateur construit à partir des pratiques de préparation et de désinfection des mamelles, variant de 0 à 13 : lavage (pas ou quelques vaches $=0$, lavette collective $=2$, lavette individuelle ou douchette $=4$ ), essuyage (aucun $=0$, chiffon ou lavette essorée collectifs $=2$, papier à usage unique $=4$ ), désinfection après la traite (aucune $=0$, trempage ou pulvérisation $=2$ ), nettoyage des mains du trayeur (pas de lavage $=0$, lavage sans désinfectant $=1$, avec désinfectant $=2$ ), tenue vestimentaire du trayeur (pas de tenue spéciale pour la traite $=0$, tenue particulière $=1$ ).

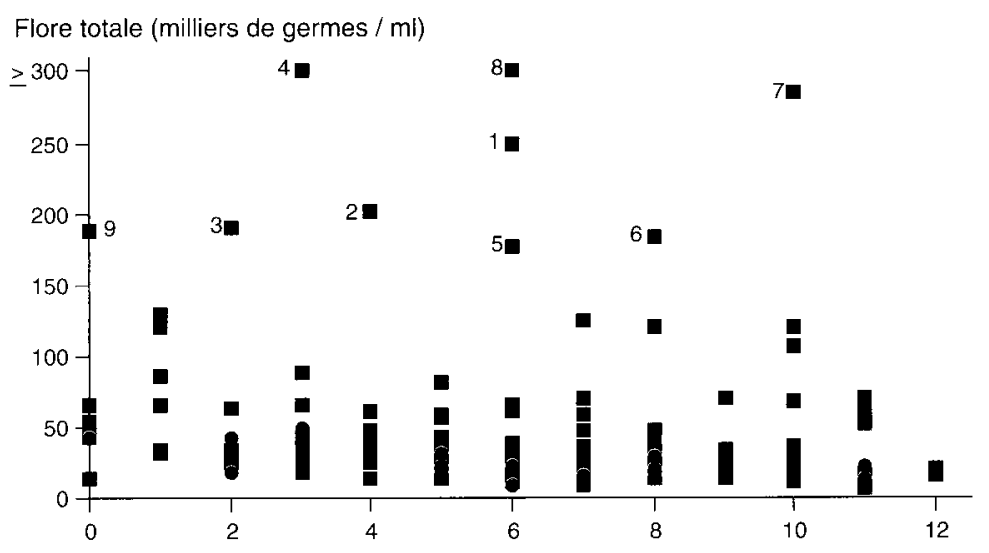

Cellutes (milliers / ml)

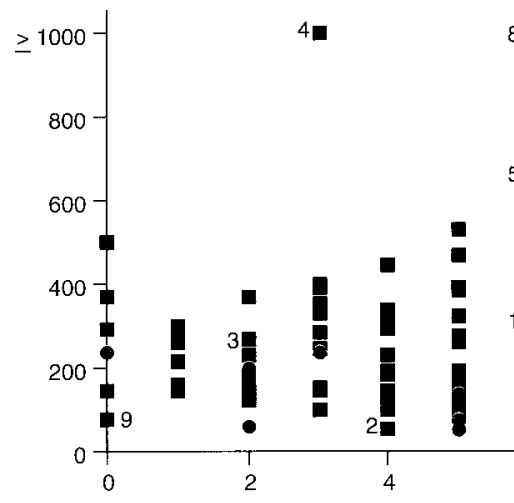

Butyriques (spores /1)

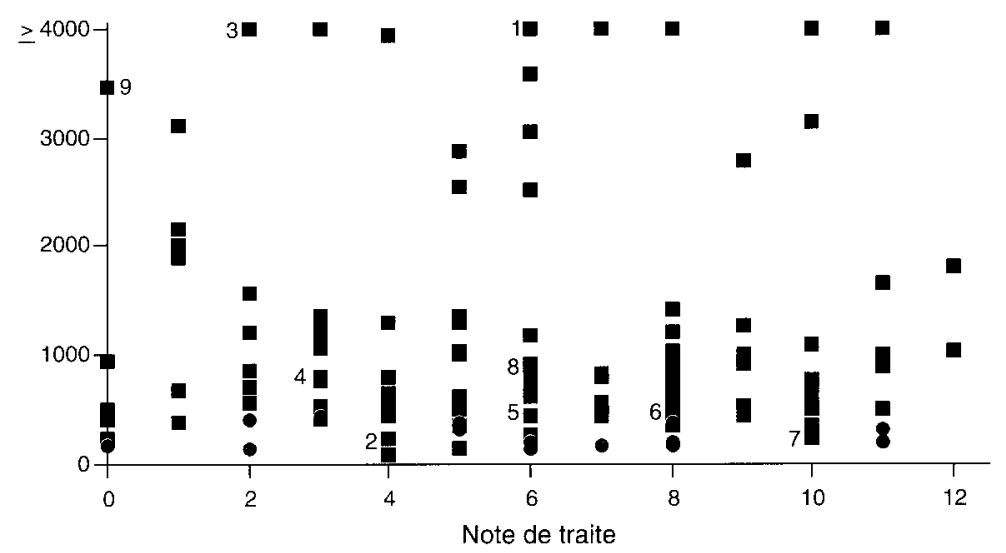

Cette figure montre que les notes de traites élevées (9 ou plus) sont associées à une numération cellulaire faible mais pas forcément à une flore totale ou à une teneur en spores butyriques faibles. Elle montre aussi que pour des notes de traite faibles, on observe à la fois des laits propres (c'est le cas de la majorité des 15 exploitations décrites en détail dans le texte et représentées en rouge sur la figure) ou très contaminés. Ces derniers ne présentent pas toujours des numérations élevées à la fois en spores butyriques, en cellules et en flore totale. Ainsi, parmi les 9 exploitations présentant une flore totale supérieure à $150000 / \mathrm{ml}$ (numérotées de 1 à 9 sur la figure), seules 3 présentent aussi une contamination en spores butyriques très élevée et 3 autres une numération cellulaire supérieure à 400 000/ml. De même, pour les 19 exploitations présentant une teneur en spores butyriques supérieure à $2500 /, 3$ seulement présentent une flore totale supérieure à $150000 / \mathrm{ml}$ et une seule une numération cellulaire supérieure à $400000 / \mathrm{ml}$. 


\title{
Références bibliographiques
}

Agabriel C., Coulon J.B., Marty G., Cheneau N., 1990. Facteurs de variation du taux protéique du lait de vache. Etude dans les exploitations du Puyde-Dôme. INRA Prod. Anim., 3, 137-150.

Agabriel C., Coulon J.B., Marty M., Bonaiti B., Boniface P., 1993. Effets respectifs de la génétique et du milieu sur la production et la composition du lait de vache. Etude en exploitations. INRA Prod. Anim., $6,213-223$.

Badinand F., 1994. Maîtrise du taux cellulaire du lait. Rec. Méd. Vét., 170, 419-427.

Ballot N., Lilas J.P., 1994. Au cœur du Pôle Fromager AOC Massif-Central, un groupement d'Intérêt Scientifique actif. Rev. ENIL, 182, 27-29.

Coulon J.B., 1991. Facteurs de variation du taux protéique du lait de vache en exploitation. Réflexions à partir de résultats d'enquêtes. INRA Prod. Anim., 4, 303-309.

Coulon J.B., Binet M., 1987. Facteurs de variation du taux protéique du lait de vache en exploitation. Etude dans l'aire de ramassage de la coopérative fromagère de Laguiole (Aveyron). Bull. Tech. CRZV Theix, INRA, 68, 11-18.

Coulon J.B., Lilas J.P., 1988. Composition chimique et contamination butyrique du lait : facteurs de variation dans le département de la Haute-Loire. INRA Prod. Anim., 1, 201-207.

Coulon J.B., Roybin D., Congy E., Garret A., 1988. Composition chimique et temps de coagulation du lait de vache : facteurs de variations dans les exploitations du Pays de Thônes. INRA Prod. Anim., 1, 253-263.

Coulon J.B., Varignier M., Darne D., 1991. Contamination butyrique du lait de vache: étude dans les exploitations de Haute-Loire. INRA Prod. Anim., 4, 369-372.

Coulon J.B., Pradel P., Verdier I., 1995. Effect of forage type on milk yield, chemical composition and clotting properties of milk. Lait, à paraitre.
Demarquilly C., Andrieu J., 1988. Les fourrages. In : R. Jarrige (ed), Alimentation des bovins, ovins et caprins, 315-335. INRA, Paris.

Dobbelaar P., Scholl D.T., Roosendaal E.A.M., Schukken Y.H., Brand A., 1994. Nutritionnal factors related to farm milk protein content - an observational study. Livest. Prod. Sci., 39, 253-262.

Dubeuf B., 1995. Relations entre les caractéristiques des laits de troupeaux, les pratiques d'élevage et les systèmes d'exploitation dans la zone de production du Beaufort. INRA Prod. Anim., 8, 105-116.

GVL, 1993. Objectif taux protéique. Références taux protéique Auvergne. GVL Massif Central, ClermontFd.

Hartheiser M., 1994. La maîtrise de la contamination du lait par les spores butyriques. Rec. Méd. Vét., 170, 429-436.

Hoden A., Coulon J.B., 1991. Maîtrise de la composition du lait : influence des facteurs nutritionnels sur la quantité et les taux de matières grasses et protéiques. INRA Prod. Anim., 4, 361-367.

Journet M., Chilliard Y., 1985. Influence de l'alimentation sur la composition chimique du lait de vache. 1. Taux butyreux : facteurs généraux. Bull. Tech. CRZV Theix, INRA, 60, 13-23.

Monsallier G., 1994. Maîtrise de la teneur en germes mésophiles totaux du lait à la production. Rec. Méd. Vét., 170, 411-418.

Rémond B., 1985. Influence de l'alimentation sur la composition chimique du lait de vache. 2 . Taux protéique : facteurs généraux. Bull. Tech. CRZV Theix, INRA, 62, 53-67.

Seegers H., Blain J.J., Lebras C., 1989. Variations du taux protéique du lait de vache. Facteurs associés aux écarts entre exploitations en région Pays de Loire. Rec. Méd. Vét., 165, 897-890.

Serieys F., 1992. Les mammites des vaches laitières. Collection "Le point sur ", Institut de l'Elevage, Paris.

\section{A survey on milk quality variations in farms.}

One hundred and fourty three dairy farms located in Massif Central were included in a detailed survey involving the herd and the farm structure, winter and summer feeding practices, milking and hygiene practices, and cows characteristics and management (breed, calving distribution...). These data allowed analysis of the variations of milk characteristics (fat and protein content, somatic cell count, total bacterial count, butyric spore count). Milk chemical composition differences among farms were higher in winter than
\end{abstract}

Abstract

in summer, and resulted essentially from breed and feeding practices (nature of the forages and nutritive supplies). Milk bacterial quality resulted both from the nature of winter forages and milking hygiene. In most of the farms, somatic cell count was higher in summer than in winter. Few farms had both a high protein content and a high milk hygienic quality.

AGABRIEL C., COULON J.B., BRUNSCHWIG G., SIBRA C., NAFIDI C., 1995. Relations entre la qualité du lait livré et les caractéristiques des exploitations. INRA Prod. Anim., 8 (4), 251-258. 